УДК 517.957

\title{
The Critical Curves of a Doubly Nonlinear Parabolic Equation in Non-divergent form with a Source and Nonlinear Boundary Flux
}

\author{
Mersaid M. Aripov* \\ National University of Uzbekistan \\ University, 4, Tashkent, 100174 \\ Uzbekistan \\ Jakhongir R. Raimbekov ${ }^{\dagger}$ \\ Inha University in Tashkent \\ Ziyolilar, 9, Tashkent, 100041 \\ Uzbekistan
}

Received 04.08.2018, received in revised form 19.09.2018, accepted 20.10.2018

The critical curves of a doubly nonlinear parabolic equation in non-divergent form with a source and nonlinear boundary flux are considered in the paper. The critical global existence curve and the critical Fujita curve are obtained.

Keywords: doubly nonlinear parabolic equation in non-divergence form, critical Fujita curve, critical global existence curve.

DOI: $10.17516 / 1997-1397-2019-12-1-112-124$.

\section{Introduction and main results}

This work studies the critical curves of a degenerate parabolic equation with doubly nonlinearity in non-divergence form with a source

$$
\frac{\partial u}{\partial t}=u^{k} \frac{\partial}{\partial x}\left(u^{m-1}\left|\frac{\partial u}{\partial x}\right|^{p-2} \frac{\partial u}{\partial x}\right)+u^{\beta}, \quad(x, t) \in \mathrm{R}_{+} \times(0 ;+\infty) .
$$

Initial condition and nonlinear boundary condition are

$$
\begin{gathered}
-u^{m-1}\left|\frac{\partial u}{\partial x}\right|^{p-2} \frac{\partial u}{\partial x}(0, t)=u^{q}(0, t), \quad t \in(0 ;+\infty), \\
u(x, 0)=u_{0}(x), \quad x \in \mathrm{R}_{+}
\end{gathered}
$$

where $p>2, m \geqslant 1, q, \beta>0,0 \leqslant k<1$ are given parameters and $u_{0}(x)$ is bounded, nontrivial, nonnegative and continuous function.

Particular case of equation (1) is a classical reaction-diffusion equation that arises in many applications like population dynamics, chemical reactions, heat transfer, etc. (see, for example,

\footnotetext{
*mirsaidaripov@mail.ru

${ }^{\dagger}$ raimbekov.jahongir@mail.ru

(C) Siberian Federal University. All rights reserved
} 
$[1,2]$ and the references therein). Equation (1) is also a model of heat propagation with gradientdependent thermal conductivity in a medium with chemical reaction and with nonlinear radiation law at the boundary [3].

Distinctive feature of equation (1) is that it degenerates at points where $u=0$ and $\nabla u=0$. Thus, it may not possess classical solutions so we consider only weak solutions in the following generalized sense.

Definition 1. A nonnegative function $u(x, t)$ is called a weak solution to problem (1)-(3) if $u$ satisfies the following conditions

1) $u \in L^{\infty}\left(\Omega_{T}\right), u_{t} \in L^{1}\left(\Omega_{T}\right), \nabla u \in L_{l o c}^{p}\left(\Omega_{T}\right)$ where $\Omega_{T}=\mathrm{R}_{+} \times(0, T)$,

2) $\int_{0}^{T} \int_{\Omega}\left\{u_{t} \varphi-u^{m-1}|\nabla u|^{p-1} \nabla u \nabla\left(u^{k} \varphi\right)-u^{\beta} \varphi\right\} d x d t+\int_{\Omega} u_{0} \varphi(x, 0)=0$,

3) $u_{0}(x) \geqslant 0$

for all test functions $\varphi \in L_{0}^{1}\left(\Omega_{T}\right)$ provided that $\varphi \geqslant 0$.

Definition 2. Let $T<\infty$ be the largest time of existence of a solution $u$ such that $\|u\|_{\infty, \Omega} \rightarrow \infty$ at $t \rightarrow T$. Then we say that solution $u(x, t)$ blows up in finite time. Otherwise, if $T=\infty$ we say that solution is global.

Conditions of global solvability and the blow up depend on coefficient $\beta$ and the gradientdependant diffusion in equation (1).

Problem (1)-(3) in divergence case $(k=0)$ have been intensively studied by many authors (see [4-11] and the references therein). Let us give some examples.

A global solution of a reaction diffusion problem with multiple nonlinearities

$$
\begin{aligned}
& u_{t}=\nabla\left(u^{m}\right)+u^{p}, \quad(x, t) \in \Omega \times(0 ; T), \\
& \frac{\partial u}{\partial \eta}=u^{q}, \quad(x, t) \in \partial \Omega \times(0 ; T), \\
& u(x, 0)=u_{0}(x), \quad x \in \Omega
\end{aligned}
$$

in a bounded domain $\Omega \subset \mathrm{R}^{N}$ was investigated by Song and Zheng [9]. They showed that the solution of the problem is global if $p, q \leqslant 0$ at $m>1$ and $p \leqslant 1, q \leqslant \frac{2 m}{m+1}$ at $m \leqslant 1$.

The critical curves for problem (1)-(3) in the case $k=0$ and $m=1$ was obtained [4].

Since $0<m<1$, the introduction of new variable $v(x, t):=(1-k)^{\frac{1-k}{k}} u^{1-k}(x, t)$ transforms equation (1) into a non-Newtonian polytropic filtration equation in divergent form. The exponents of the equation were studied [12].

The critical exponents for a p-Laplacian equation with variable density and source were obtained [6]. The results of this work are given below. Any solution is global at $q, \beta>0$ from the region $R_{g}=\left\{0<\beta \leqslant 1,0<q \leqslant \frac{(2-n)(p-1)}{p-n}\right\} \cup\left\{\beta>\frac{(p-n) q-p+1}{p-1}, q>(2-n)(p-1)\right\}$. Any solution blows up when parameters are in the region $R_{g}^{\prime}$.

The degenerate parabolic equations in non-divergent form were considered by many authors (see [13-18] and the references therein). Let us give a couple of examples.

Some qualitative properties of a p-Laplacian equation in non-divergent form were studied by Raimbekov [13]. Asymptotic solution of the Cauchy problem was also analysed at $t \rightarrow \infty$ for slow and fast diffusion cases. 
Jin and Yin [14] considered the doubly degenerate diffusion Cauchy problem

$$
\begin{gathered}
u_{t}=u^{m} \operatorname{div}\left(|\nabla u|^{(p-2)} \nabla u\right)+\lambda u^{q}, \quad(x, t) \in \Omega \times(0 ; T), \\
u=0, \quad(x, t) \in \partial \Omega \times(0 ; T), \\
u(x, 0)=u_{0}(x), \quad x \in \Omega,
\end{gathered}
$$

where $m>1, p>1$. The critical exponent $q_{c}=p+m-1$ was obtained, namely, the solutions are global if $q<q_{c}$, and there exist both global and blow-up solutions if $q>q_{c}$. In the critical case $q=q_{c}$ solutions are dictated by the size of the domain.

The main objective of this work is to define the critical curves and the region of parameters where global solutions and blow-up solutions exist. The results of the present work are the following theorems.

\section{Theorem 1.}

(i) In the region of parameters

$$
G=\left\{0<\beta \leqslant 1,0<q \leqslant \frac{p(1-k)+(k+m+p-3)}{p}\right\}
$$

any solution of problem (1)-(3) is global in time.

(ii) In the region

$$
R=\{\beta>1\} \cup\left\{q>\frac{p(1-k)+(k+m+p-3)}{p}\right\}
$$

there exist solutions of (1)-(3) that blow up in finite time.

Remark 1. Theorem 1 defines the critical global existence curve of problem (1)-(3) as $\left\{\beta=1,0<q \leqslant \frac{p(1-k)+(k+m+p-3)}{p}\right\} \cup\left\{0<\beta \leqslant 1, q=\frac{p(1-k)+(k+m+p-3)}{p}\right\}$ (see Fig. 1).

\section{Theorem 2.}

(i) In the region $R_{u} \subset R$

$$
\begin{aligned}
R_{u}= & \{\beta>1, q \leqslant p(1-k)+(k+m+p-3)\} \cup \\
& \cup\left\{\beta \leqslant p(1-k)+k+m+p-2, q>\frac{p(1-k)+(k+m+p-3)}{p}\right\},
\end{aligned}
$$

any solution of problem (1)-(3) blows up in finite time.

(ii) In the region $R_{g} \subset R$

$$
R_{g}=\{\beta>p(1-k)+k+m+p-2, q>p(1-k)+(k+m+p-3)\},
$$

there exist both global and blow-up solutions of problem (1)-(3).

Remark 2. Theorem 1 defines the critical Fujita curve of problem (1)-(3). It is given by $\{\beta=p(1-k)+k+m+p-2, q \geqslant p(1-k)+(k+m+p-3)\} \cup\{\beta \geqslant p(1-k)+k+m+p-2, q=$ $=p(1-k)+(k+m+p-3)\}$ (see Fig. 1$)$.

In what follows we give proofs of theorems. 


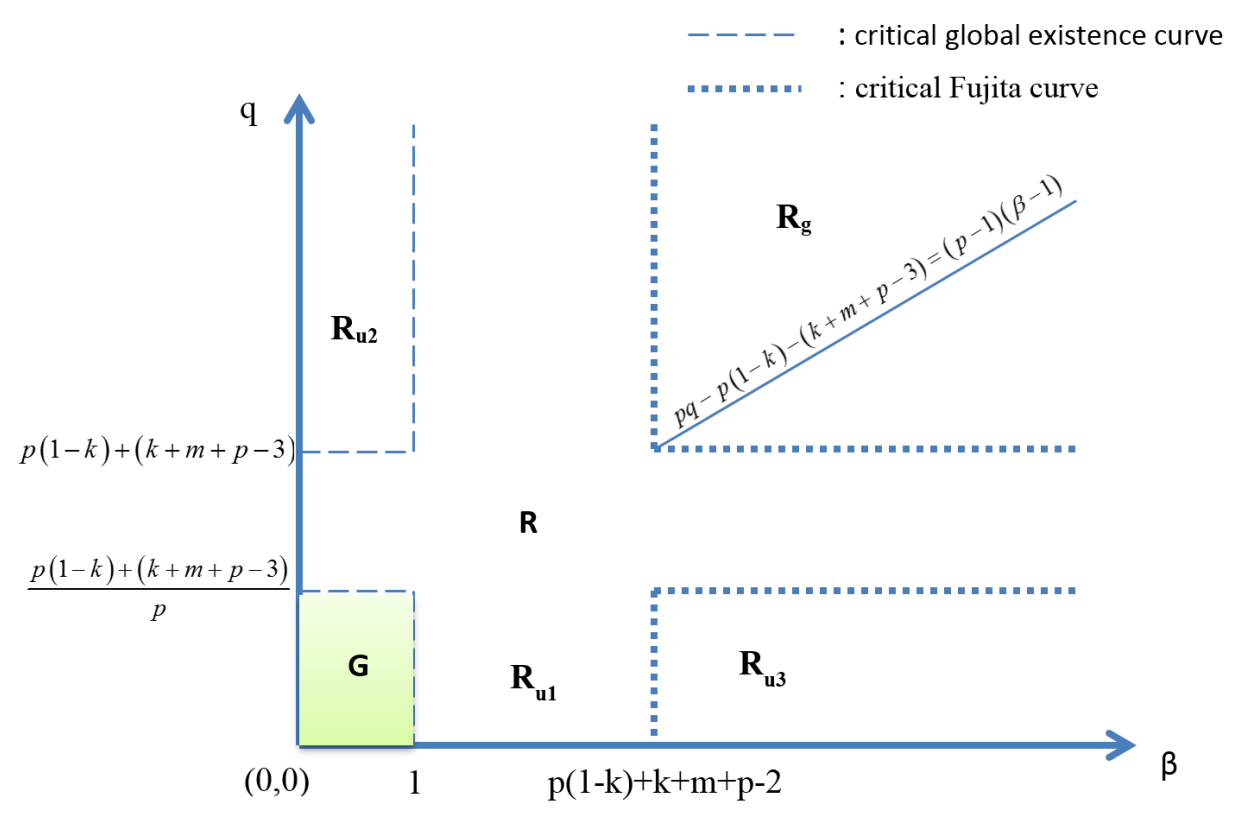

Fig. 1. Critical curves

\section{Critical global existence curve}

Proof of Theorem 1(i). Let us consider the following globally defined in time supersolution in the self-similar form

$$
\bar{u}(t, x)=e^{L t}\left(K+e^{-M \xi}\right), \xi=x e^{-J t}
$$

with parameters $K>\left\|u_{0}\right\|_{\infty}, M \geqslant(K+1)^{\frac{q+1-m}{p-1}}, L \geqslant \frac{M^{p}(m+p-2)(K+1)^{k+m-1}+(K+1)^{\beta}}{K}$ and $J=\frac{k+m+p-3}{p} L$.

One can obtain that

$$
\begin{gathered}
\frac{\partial \bar{u}}{\partial t}=L e^{L t}\left(K+e^{-M \xi}\right)+J M x e^{-J t+L t-M \xi} \geqslant L e^{L t}\left(K+e^{-M \xi}\right) \geqslant K L e^{L t}, \\
\bar{u}^{\beta}=e^{\beta L t}\left(K+e^{-M \xi}\right)^{\beta} \leqslant e^{\beta L t}(K+1)^{\beta}, \\
\frac{\partial \bar{u}}{\partial x}=-M e^{(L-J) t} e^{-M \xi}, \\
u^{k} \frac{\partial}{\partial x}\left(u^{m-1}\left|\frac{\partial u}{\partial x}\right|^{p-2} \frac{\partial u}{\partial x}\right)=M^{p}(m-1) e^{k L t}\left(K+e^{-M \xi}\right)^{k+m-2} e^{(m+p-2) L t-J p t-M p \xi}+ \\
+M^{p}(p-1) e^{k L t}\left(K+e^{-M \xi}\right)^{k+m-1} e^{(m+p-2) L t-J p t-M(p-1) \xi} \leqslant \\
\leqslant M^{p}(m+p-2)(K+1)^{k+m-1} e^{(k+m+p-2) L t-J p t-M(p-1) \xi},
\end{gathered}
$$

and we have on the boundary

$$
-u^{m-1}\left|\frac{\partial u}{\partial x}\right|^{p-2} \frac{\partial u}{\partial x}(0, t)=(K+1)^{m-1} M^{p-1} e^{(m+p-2) L t-J(p-1) t} .
$$

By the definition of $K, M, L, J$ and the assumption $(\beta, q) \in G$ we have 


$$
\begin{aligned}
\frac{\partial \bar{u}}{\partial t} \geqslant \bar{u}^{k} \frac{\partial}{\partial x}\left(\bar{u}^{m-1}\left|\frac{\partial \bar{u}}{\partial x}\right|^{p-2} \frac{\partial \bar{u}}{\partial x}\right)+\bar{u}^{\beta}, \quad(x, t) \in \mathrm{R}_{+} \times \mathrm{R}_{+}, \\
-\bar{u}^{m-1}\left|\frac{\partial \bar{u}}{\partial x}\right|^{p-2} \frac{\partial \bar{u}}{\partial x}(0, t) \geqslant \bar{u}^{q}(0, t), \quad t \in(0 ;+\infty) .
\end{aligned}
$$

Thus, $\bar{u}$ is a supersolution of problem (1)-(3). It implies that every solution of problem (1)-(3) is global solution provided $(\beta, q) \in G$.

Proof of Theorem 1 (ii). Let us consider a blow-up solution of the Cauchy problem

$$
\begin{aligned}
& \frac{\partial u}{\partial t}=u^{k} \frac{\partial}{\partial x}\left(u^{m-1}\left|\frac{\partial u}{\partial x}\right|^{p-2} \frac{\partial u}{\partial x}\right)+u^{\beta}, \quad(x, t) \in \mathrm{R} \times(0 ;+\infty), \\
& u(0, t)=u_{0}(x),
\end{aligned}
$$

where $\beta>1$. It is symmetric and satisfies

$$
\begin{aligned}
& \frac{\partial u}{\partial t}=u^{k} \frac{\partial}{\partial x}\left(u^{m-1}\left|\frac{\partial u}{\partial x}\right|^{p-2} \frac{\partial u}{\partial x}\right)+u^{\beta}, \quad(x, t) \in \mathrm{R}_{+} \times(0, T), \\
& -u^{m-1}\left|\frac{\partial u}{\partial x}\right|^{p-2} \frac{\partial u}{\partial x}(0, t)=0, \quad t \in(0, T), \\
& u(x, 0)=u_{0}(x) .
\end{aligned}
$$

It can be shown that solutions of problem (5) are always subsolutions of problem (1)-(3) for the same initial value. It is known [14] that solutions of problem (5) for sufficiently large $u_{0}$ blow up in a finite time interval. It follows from the comparison principle that solution of problem (1)-(3) also blows up.

If $q>\frac{p(1-k)+(k+m+p-3)}{p}$ then it follows from [7] that the problem

$$
\begin{aligned}
& \frac{\partial u}{\partial t}=u^{k} \frac{\partial}{\partial x}\left(u^{m-1}\left|\frac{\partial u}{\partial x}\right|^{p-2} \frac{\partial u}{\partial x}\right), \quad(x, t) \in \mathrm{R}_{+} \times(0,+\infty), \\
& -u^{m-1}\left|\frac{\partial u}{\partial x}\right|^{p-2} \frac{\partial u}{\partial x}(0, t)=u^{q}(0, t), \quad t \in(0,+\infty), \\
& u(x, 0)=u_{0}(x)
\end{aligned}
$$

has a solution that blows up. We use them as subsolutions of problem (1)-(3). The result immediately follows from the comparison principle.

\section{Critical Fujita curve}

Proof of Theorem 2(i). Let us introduce the following designations

$$
\begin{aligned}
& R_{u 1}=\{1<\beta \leqslant p(1-k)+k+m+p-2\} \cup \\
& \cup\left\{\frac{p(1-k)+(k+m+p-3)}{p}<q \leqslant p(1-k)+(k+m+p-3)\right\}, \\
& R_{u 2}=\{\beta \leqslant 1, q>p(1-k)+(k+m+p-3)\}, \\
& R_{u 3}=\left\{\beta>p(1-k)+(k+m+p-2), q \leqslant \frac{p(1-k)+(k+m+p-3)}{p}\right\} .
\end{aligned}
$$


Because $R_{u}=R_{u 1} \cup R_{u 2} \cup R_{u 3}$ (see Fig. 1) the proof is divided into three parts.

Case 1. $(\beta, q) \in R_{u 1}$. Since each solution of problem (5) with nontrivial initial value blows up if $1<\beta \leqslant p(1-k)+k+m+p-2$ (see [14]) and every solution of problem (6) with nontrivial data also blows up when $\frac{p(1-k)+(k+m+p-3)}{p}<q \leqslant p(1-k)+(k+m+p-3)$ (see [7]) we can consider them as subsolutions of problem (1)-(3). The result immediately follows from the comparison principle.

Case 2. $(\beta, q) \in R_{u 2}$. Let $\beta<1$ and the following self-similar subsolution of problem (5) has the form

$$
\underline{u}(x, t)=t^{\alpha} \phi(\xi), \quad \xi=x t^{-\gamma},
$$

where $\alpha=\frac{1}{1-\beta}, \gamma=\frac{k+m+p-2-\beta}{p(1-\beta)}$.

One can obtain that

$$
\begin{aligned}
& \frac{\partial \underline{u}}{\partial t}=t^{\alpha-1}\left(\alpha \phi(\xi)-\gamma \xi \phi^{\prime}(\xi)\right), \\
& \underline{u}^{m-1}\left|\frac{\partial \underline{u}}{\partial x}\right|^{p-2} \frac{\partial \underline{u}}{\partial x}=t^{(p-1)(\alpha-\gamma)+\alpha(m-1)} \phi^{m-1}\left|\phi^{\prime}\right|^{p-2} \phi^{\prime}(\xi), \\
& \underline{u}^{k} \frac{\partial}{\partial x}\left(\underline{u}^{m-1}\left|\frac{\partial \underline{u}}{\partial x}\right|^{p-2} \frac{\partial \underline{u}}{\partial x}\right)=t^{(k+m+p-2) \alpha-p \gamma} \phi^{k}\left(\phi^{m-1}\left|\phi^{\prime}\right|^{p-2} \phi^{\prime}\right)^{\prime}(\xi) .
\end{aligned}
$$

Then we have

$$
\begin{aligned}
& \frac{\partial \underline{u}}{\partial t} \leqslant \underline{u}^{k} \frac{\partial}{\partial x}\left(\underline{u}^{m-1}\left|\frac{\partial \underline{u}}{\partial x}\right|^{p-2} \frac{\partial \underline{u}}{\partial x}\right), \quad(x, t) \in \mathrm{R}_{+} \times(0,+\infty), \\
& \underline{u}^{m-1}\left|\frac{\partial \underline{u}}{\partial x}\right|^{p-2} \frac{\partial \underline{u}}{\partial x}(0, t)=0, \quad t \in(0,+\infty)
\end{aligned}
$$

if $\phi(\xi)$ satisfies

$$
\begin{aligned}
& \phi^{k}\left(\phi^{m-1}\left|\phi^{\prime}\right|^{p-2} \phi^{\prime}\right)^{\prime}(\xi)+\gamma \xi \phi^{\prime}(\xi)+\phi^{\beta}(\xi)-\alpha \phi(\xi) \geqslant 0 \\
& -\phi^{m-1}\left|\phi^{\prime}\right|^{p-2} \phi^{\prime}(0)=0 .
\end{aligned}
$$

Let us define

$$
\phi(\xi)=A\left(a-\xi^{\frac{p}{p-1}}\right)_{+}^{\frac{p-1}{k+m+p-3}},
$$

where parameters $a, A$ are to be determined. One can obtain that

$$
\begin{aligned}
& \phi^{\prime}(\xi)=-A \frac{p}{k+m+p-3}\left(a-\xi^{\frac{p}{p-1}}\right)_{+}^{\frac{p-1}{k+m+p-3}-1} \xi^{\frac{1}{p-1}}, \\
& \phi^{m-1}\left|\phi^{\prime}\right|^{p-2} \phi^{\prime}(\xi)=-A^{m+p-2}\left(\frac{p}{k+m+p-3}\right)^{p-1}\left(a-\xi^{\frac{p}{p-1}}\right)_{+}^{\frac{(p-1)(1-k)}{k+m+p-3}} \xi, \\
& \phi^{k}\left(\phi^{m-1}\left|\phi^{\prime}\right|^{p-2} \phi^{\prime}\right)^{\prime}(\xi)=A^{k+m+p-2}\left(\frac{p}{k+m+p-3}\right)^{p}(1-k)\left(a-\xi^{\frac{p}{p-1}}\right)_{+}^{\frac{p-1}{k+m+p-3}-1} \xi^{\frac{p}{p-1}}- \\
& -A^{k+m+p-2}\left(\frac{p}{k+m+p-3}\right)^{p-1}\left(a-\xi^{\frac{p}{p-1}}\right)_{+}^{\frac{p-1}{k+m+p-3}} \text {. }
\end{aligned}
$$


Since $\beta<1$ we get

$$
\begin{aligned}
& \phi^{\beta}(\xi)=A^{\beta}\left(a-\xi^{\frac{p}{p-1}}\right)_{+}^{\frac{\beta(p-1)}{k+m+p-3}}= \\
& =A^{\beta}\left(a-\xi^{\frac{p}{p-1}}\right)_{+}^{\frac{p-1}{k+m+p-3}}\left(a-\xi^{\frac{p}{p-1}}\right)_{+}^{\frac{(\beta-1)(p-1)}{k+m+p-3}}>A^{\beta} a^{\frac{(\beta-1)(p-1)}{k+m+p-3}}\left(a-\xi^{\frac{p}{p-1}}\right)_{+}^{\frac{p-1}{k+m+p-3}} .
\end{aligned}
$$

Assuming that

$$
\begin{gathered}
A^{k+m+p-3}\left(\frac{p}{k+m+p-3}\right)^{p-1} \geqslant \gamma, \\
a^{\frac{(\beta-1)(p-1)}{k+m+p-3}} \geqslant A^{k+m+p-2-\beta}\left(\frac{p}{k+m+p-3}\right)^{p-1}+A^{1-\beta} \alpha,
\end{gathered}
$$

we can verify that (9) holds. The subsolution $\underline{u}$ defined in (7) and (10) has zero initial value. Hence it is a subsolution of problem (5) with any nontrivial initial data.

If $\beta=1$ we construct a compact support self-similar solution to problem (5) of the form

$$
U(x, t)=e^{\alpha(t-\tau)} \phi(\xi), \quad \xi=x e^{-\gamma(t-\tau)},
$$

where $\alpha=\frac{(1-k) p}{(k+m+p-3)+(1-k) p}, \quad \gamma=\frac{(1-k)(k+m+p-3)}{(k+m+p-3)+(1-k) p}$ and

$$
\phi(\xi)=\left(\frac{k+m+p-3}{p}\right)^{\frac{p-1}{k+m+p-3}}\left[\frac{(k+m+p-3)}{(k+m+p-3)+(1-k) p}\right]^{\frac{1}{k+m+p-3}}\left(a-\xi^{\frac{p}{p-1}}\right)^{\frac{p-1}{k+m+p-3}}
$$

One can obtain that

$$
\begin{gathered}
\frac{\partial U}{\partial t}=e^{\alpha(t-\tau)}\left(\alpha \phi(\xi)-\gamma \xi \phi^{\prime}(\xi)\right) \\
U^{m-1}\left|\frac{\partial U}{\partial x}\right|^{p-2} \frac{\partial U}{\partial x}=e^{\alpha(m+p-2)(t-\tau)-\gamma(p-1)(t-\tau)} \phi^{m-1}|\phi|^{p-2} \phi^{\prime}(\xi), \\
U^{k} \frac{\partial}{\partial x}\left(U^{m-1}\left|\frac{\partial U}{\partial x}\right|^{p-2} \frac{\partial U}{\partial x}\right)=e^{[\alpha(k+m+p-2)-\gamma p](t-\tau)} \phi^{k}\left(\phi^{m-1}|\phi|^{p-2} \phi^{\prime}\right)^{\prime}(\xi) .
\end{gathered}
$$

It follows from equalities given above and (11) that

$$
\begin{gathered}
U^{k} \frac{\partial}{\partial x}\left(U^{m-1}\left|\frac{\partial U}{\partial x}\right|^{p-2} \frac{\partial U}{\partial x}\right)+U=0, \quad(x, t) \in \mathrm{R}^{+} \times(0, T), \\
-U^{m-1}\left|\frac{\partial U}{\partial x}\right|^{p-2} \frac{\partial U}{\partial x}(0, t)=0, \quad t \in(0, T) .
\end{gathered}
$$

If $\tau \geqslant 0$ is large such that $U(x, 0) \leqslant u_{0}(x)$ then $U(x, t)$ is a subsolution of problem (5). Any subsolution of problem (5) is also a subsolution of problem (1)-(3) with the same initial data. Thus there exists a finite time $t_{0}$ such that solution of problem (1)-(3) is greater than the initial value of the blow-up subsolution of (6)

$$
\nu(x, t)=(T-t)^{-s} \psi(\xi), \quad \xi=x(T-t)^{-l},
$$

where $l=\frac{(q+2-p-m)}{p(q+k-1)-(k+p+m-3)}, s=\frac{(p-1)}{p(q+k-1)-(k+p+m-3)}$, and

$$
\psi(\xi)=B\left(d-\xi^{\frac{p}{p-1}}\right)^{\frac{p-1}{k+m+p-3}} .
$$


Parameters $B$ and $d$ are some positive constants [7]. Note that $\nu$ is a subsolution of problem (6) and it is also a strict subsolution of problem (1)-(3). Hence, the solution of problem (1) $-(3)$ blows up in a finite time $t \leqslant T+t_{0}$.

Case 3. $(\beta, q) \in R_{u 3}$. If $p q \leqslant p(1-k)+(k+m+p-3)$ we consider the following self-similar subsolution of problem (5) in the form

$$
\underline{u}(t, x)=t^{\alpha} \phi(\xi), \quad \xi=x t^{-\gamma},
$$

where $\gamma=\frac{m+p-2-q}{(k+p+m-3)+p(1-k-q)}, \alpha=\frac{p-1}{(k+p+m-3)+p(1-k-q)}$.

We obtain from (8) that

$$
\begin{aligned}
& \frac{\partial \underline{u}}{\partial t} \leqslant \underline{u}^{k} \frac{\partial}{\partial x}\left(\underline{u}^{m-1}\left|\frac{\partial \underline{u}}{\partial x}\right|^{p-2} \frac{\partial \underline{u}}{\partial x}\right), \quad(x, t) \in \mathrm{R}_{+} \times(0,+\infty), \\
& \underline{u}^{m-1}\left|\frac{\partial \underline{u}}{\partial x}\right|^{p-2} \frac{\partial \underline{u}}{\partial x}(0, t) \leqslant \underline{u}^{q}, \quad t \in(0,+\infty),
\end{aligned}
$$

if $\phi(\xi)$ satidfies

$$
\begin{aligned}
& \phi^{k}\left(\phi^{m-1}\left|\phi^{\prime}\right|^{p-2} \phi^{\prime}\right)^{\prime}(\xi)+\gamma \xi \phi^{\prime}(\xi)+\phi^{\beta}(\xi)-\alpha \phi(\xi) \geqslant 0, \\
& -\phi^{m-1}\left|\phi^{\prime}\right|^{p-2} \phi^{\prime}(0) \leqslant \phi^{q}(0) .
\end{aligned}
$$

Then we take

$$
\phi(\xi)=C(c-\xi)_{+}^{\frac{p-1}{k+m+p-3}},
$$

where $C, c$ are constants to be determined. It is easy to see that

$$
\begin{gathered}
\phi^{\prime}(\xi)=-C \frac{p-1}{k+m+p-3}(c-\xi)_{+}^{\frac{p-1}{k+m+p-3}-1} \\
\phi^{m-1}\left|\phi^{\prime}\right|^{p-2} \phi^{\prime}(\xi)=-C^{m+p-2}\left(\frac{p-1}{k+m+p-3}\right)^{p-1}(c-\xi)_{+}^{\frac{(p-1)(1-k)}{k+m+p-3}} \\
\phi^{k}\left(\phi^{m-1}\left|\phi^{\prime}\right|^{p-2} \phi^{\prime}\right)^{\prime}(\xi)=C^{k+m+p-2}(1-k)\left(\frac{p-1}{k+m+p-3}\right)^{p}(c-\xi)_{+}^{\frac{(p-1)}{k+m+p-3}-1} .
\end{gathered}
$$

Taking into account that

$$
\begin{gathered}
\gamma \xi \phi^{\prime}(\xi)-\alpha \phi(\xi) \geqslant-\gamma C \frac{p-1}{k+m+p-3} c(c-\xi)_{+}^{\frac{p-1}{k+m+p-3}-1}-\alpha C c(c-\xi)_{+}^{\frac{p-1}{k+m+p-3}-1}= \\
=-C c\left(\frac{\gamma(p-1)}{k+m+p-3}+\alpha\right)(c-\xi)_{+}^{\frac{p-1}{k+m+p-3}-1}, \quad \text { for } 0 \leqslant \xi \leqslant A,
\end{gathered}
$$

and $p q \leqslant p(1-k)+(k+m+p-3)$, we can choose $C$ and $c$ such that they satisfy

$$
\begin{gathered}
C^{k+m+p-3}(1-k)\left(\frac{p-1}{k+m+p-3}\right)^{p} \geqslant\left(\frac{\gamma(p-1)}{k+m+p-3}+\alpha\right) c, \\
C^{m+p-2-q}\left(\frac{p-1}{k+m+p-3}\right)^{p-1} \leqslant c^{\frac{(p-1)(q+k-1)}{k+m+p-3}} .
\end{gathered}
$$

It is not difficult to verify that (13) holds. Subsolution (12) has zero initial value. Thus $\underline{u}$ defined in (12) and (14) is a subsolution of problem (6) with every nontrivial data. If $p q=$ 
$=p(1-k)+(k+m+p-3)$ then we also construct a self-similar subsolution to problem (6) in exponent type

$$
\underline{u}(x, t)=e^{\alpha(t-\tau)} \phi(\xi), \quad \xi=x e^{-\gamma(t-\tau)},
$$

where $\alpha=\frac{p(1-k)}{2 p-1}, \gamma=\frac{(1-k)(k+m+p-3)}{2 p-1}$. Let

$$
\phi(\xi)=C\left(C^{k+m+p-3}\left(\frac{p-1}{k+m+p-3}\right)^{p}-\xi\right)^{\frac{p-1}{k+m+p-3}} .
$$

Using (15), we obtain for arbitrary $C>0$ that

$$
\begin{gathered}
\phi^{k}\left(\phi^{m-1}\left|\phi^{\prime}\right|^{p-2} \phi^{\prime}\right)^{\prime}(\xi)+\gamma \xi \phi^{\prime}(\xi)-\alpha \phi(\xi) \geqslant 0, \quad \text { for } 0 \leqslant \xi \leqslant C^{k+m+p-3}\left(\frac{p-1}{k+m+p-3}\right)^{p}, \\
-\phi^{m-1}\left|\phi^{\prime}\right|^{p-2} \phi^{\prime}(0) \leqslant \phi^{\frac{p(1-k)+(k+m+p-3)}{p}}(0) .
\end{gathered}
$$

Using (12) and inequalities given above, we have

$$
\begin{aligned}
& \frac{\partial \underline{u}}{\partial t} \leqslant \underline{u}^{k} \frac{\partial}{\partial x}\left(\underline{u}^{m-1}\left|\frac{\partial \underline{u}}{\partial x}\right|^{p-2} \frac{\partial \underline{u}}{\partial x}\right), \quad(x, t) \in \mathrm{R}_{+} \times(0,+\infty), \\
& \underline{u}^{m-1}\left|\frac{\partial \underline{u}}{\partial x}\right|^{p-2} \frac{\partial \underline{u}}{\partial x}(0, t)=\underline{u}^{q}, \quad t \in(0,+\infty) .
\end{aligned}
$$

If we choose large $\tau \geqslant 0$ and suitable $C$ such that $\underline{u}(x, 0) \leqslant u_{0}(x)$ then $\underline{u}(x, t)$ is a subsolution of problem (6) and it is large enough. The proof follows from the results of Jin and Yin [14] where a blow-up subsolution to problem (5) is constructed.

Proof of Theorem 2 (ii). Taking into account that

$$
\begin{aligned}
R_{g}= & \{p q-p(1-k)-(k+m+p-3)<(p-1)(\beta-1), q>p(1-k)+(k+m+p-3)\} \cup \\
& \cup\{p q-p(1-k)-(k+m+p-3) \geqslant(p-1)(\beta-1), \beta>p(1-k)+(k+m+p-2)\}
\end{aligned}
$$

(see Fig. 1), we divide the proof into 2 cases.

Case 1. $p q-p(1-k)-(k+m+p-3)<(p-1)(\beta-1), q>p(1-k)+(k+m+p-3)$. Let us introduce the auxiliary function

$$
\bar{u}(x, t)=(t+\tau)^{-\alpha} f(\xi), \quad \xi=x(t+\tau)^{-\gamma}
$$

with $\tau>0$ and $\gamma=\frac{q-(m+p-2)}{p q-p(1-k)-(k+m+p-3)}, \alpha=\frac{(p-1)}{p q-p(1-k)-(k+m+p-3)}$.

One can obtain that

$$
\begin{aligned}
\frac{\partial \bar{u}}{\partial t} & =(t+\tau)^{-(\alpha+1)}\left(-\alpha f(\xi)-\gamma \xi f^{\prime}(\xi)\right), \\
\bar{u}^{m-1}\left|\frac{\partial \bar{u}}{\partial x}\right|^{p-2} \frac{\partial \bar{u}}{\partial x} & =(t+\tau)^{-(m+p-2) \alpha-(p-1) \gamma} f^{m-1}\left|f^{\prime}\right|^{p-2} f^{\prime} \\
\bar{u}^{k} \frac{\partial}{\partial x}\left(\bar{u}^{m-1}\left|\frac{\partial \bar{u}}{\partial x}\right|^{p-2} \frac{\partial \bar{u}}{\partial x}\right) & =(t+\tau)^{-(k+m+p-2) \alpha-p \gamma} f^{k}\left(f^{m-1}\left|f^{\prime}\right|^{p-2} f^{\prime}\right)^{\prime} .
\end{aligned}
$$

Therefore, $\bar{u}$ is a supersolution of problem (1)-(3) with small initial data if the function $f(\xi)$ satisfies

$$
\begin{aligned}
& f^{k}\left(f^{m-1}\left|f^{\prime}\right|^{p-2} f^{\prime}\right)^{\prime}+\alpha f+\gamma \xi f^{\prime}+(t+\tau)^{-\alpha \beta+\alpha+1} f^{\beta} \leqslant 0, \\
& f^{m-1}\left|f^{\prime}\right|^{p-2} f^{\prime}(0) \geqslant f^{q}(0) .
\end{aligned}
$$


Inequality $q>p(1-k)+(k+m+p-3)$ implies that $\frac{1}{p(1-k)+(k+m+p-3)}-\alpha>0$. Let $\varepsilon==\frac{1}{p(1-k)+(k+m+p-3)}-\alpha$ and we first consider

$$
\begin{gathered}
f^{k}\left(f^{m-1}\left|f^{\prime}\right|^{p-2} f^{\prime}\right)^{\prime}+\gamma \xi f^{\prime}+(\alpha+\varepsilon) f \leqslant 0 \\
f^{m-1}\left|f^{\prime}\right|^{p-2} f^{\prime}(0) \geqslant f^{q}(0) .
\end{gathered}
$$

Let us assume that

$$
f(\xi)=A\left((\lambda b)^{\frac{p}{p-1}}-(\xi+b)^{\frac{p}{p-1}}\right)_{+}^{\frac{p-1}{k+m+p-3}},
$$

where constants $A, \lambda>1, b>0$ are to be determined. One can obtain that

$$
\begin{aligned}
& f^{\prime}(\xi)=-A \frac{p}{k+m+p-3}\left((\lambda b)^{\frac{p}{p-1}}-(\xi+b)^{\frac{p}{p-1}}\right)_{+}^{\frac{p-1}{k+m+p-3}-1}(\xi+b)^{\frac{1}{p-1}} \\
& f^{m-1}\left|f^{\prime}\right|^{p-2} f^{\prime}(\xi)=-A^{m+p-2}\left(\frac{p}{k+m+p-3}\right)^{p-1}\left((\lambda b)^{\frac{p}{p-1}}-(\xi+b)^{\frac{p}{p-1}}\right)_{+}^{\frac{(p-1)(1-k)}{k+m+p-3}}(\xi+b) \\
& f^{k}\left(f^{m-1}\left|f^{\prime}\right|^{p-2} f^{\prime}\right)^{\prime}(\xi)= \\
& =A^{k+m+p-2}(1-k)\left(\frac{p}{k+m+p-3}\right)^{p}\left((\lambda b)^{\frac{p}{p-1}}-(\xi+b)^{\frac{p}{p-1}}\right)_{+}^{\frac{p-1}{k+m+p-3}-1}(\xi+b)^{\frac{p}{p-1}}- \\
& \quad-A^{k+m+p-2}\left(\frac{p}{k+m+p-3}\right)^{p-1}\left((\lambda b)^{\frac{p}{p-1}}-(\xi+b)^{\frac{p}{p-1}}\right)_{+}^{\frac{p-1}{k+m+p-3}} .
\end{aligned}
$$

Substituting $f$ and $f^{\prime}$ into (18), we obtain

$$
\begin{aligned}
A^{k+m+p-3} & (1-k)\left(\frac{p}{k+m+p-3}\right)^{p}(\xi+b)^{\frac{p}{p-1}}-\gamma \frac{p}{k+m+p-3} \xi(\xi+b)^{\frac{1}{p-1}}+ \\
+ & {\left[(\alpha+\varepsilon)-A^{k+m+p-3}\left(\frac{p}{k+m+p-3}\right)^{p-1}\right]\left((\lambda b)^{\frac{p}{p-1}}-(\xi+b)^{\frac{p}{p-1}}\right) \leqslant 0 }
\end{aligned}
$$

for $0 \leqslant \xi \leqslant(\lambda-1) b$,

$$
\begin{aligned}
A^{m+p-2}\left(\frac{p}{k+m+p-3}\right)^{p-1} b^{\frac{(k+m+p-3)+p(1-k)}{k+m+p-3}} & \left((\lambda)^{\frac{p}{p-1}}-1\right)_{+}^{\frac{(p-1)(1-k)}{k+m+p-3}} \geqslant \\
& \geqslant A^{q} b^{\frac{q p}{k+m+p-3}}\left(\lambda^{\frac{p}{p-1}}-1\right)_{+}^{\frac{q(p-1)}{k+m+p-3}} .
\end{aligned}
$$

Setting $\eta=\xi+b$ and taking into account that $\alpha(k+m+p-3)=1-\gamma p$, we can rewrite $(20)$ as

$$
\begin{gathered}
\frac{1}{k+m+p-3}\left[A^{k+m+p-3}((1-k) p+(k+m+p-3))\left(\frac{p}{k+m+p-3}\right)^{p-1}-1-\right. \\
-(k+m+p-3) \varepsilon] \eta^{\frac{p}{p-1}}++\frac{\gamma p}{k+m+p-3} b \eta^{\frac{1}{p-1}}- \\
-\left[A^{k+m+p-3}\left(\frac{p}{k+m+p-3}\right)^{p-1}-(\alpha+\varepsilon)\right](\lambda b)^{\frac{p}{p-1}} \leqslant 0, \quad b \leqslant \eta \leqslant \lambda b .
\end{gathered}
$$

For simplicity we introduce the following designation 


$$
\begin{aligned}
g(y) & =\left[A^{k+m+p-3}((1-k) p+(k+m+p-3))\left(\frac{p}{k+m+p-3}\right)^{p-1}-1-(k+m+p-3) \varepsilon\right] \times \\
& \times \frac{y^{p}}{k+m+p-3}+\frac{\gamma p}{k+m+p-3} b y-\left[A^{k+m+p-3}\left(\frac{p}{k+m+p-3}\right)^{p-1}-(\alpha+\varepsilon)\right](\lambda b)^{\frac{p}{p-1}} .
\end{aligned}
$$

Then we have

$$
\begin{aligned}
g^{\prime}(y)=\frac{\gamma p}{k+m+p-3} b+\frac{p}{k+m+p-3}\left[A^{k+m+p-3}((1-k) p+\right. \\
\left.\quad+(k+m+p-3))\left(\frac{p}{k+m+p-3}\right)^{p-1}-1-(k+m+p-3) \varepsilon\right] y^{p-1} .
\end{aligned}
$$

Therefore, to prove $(22)$ one need to prove that $g\left(\eta^{\frac{1}{p-1}}\right) \leqslant 0$ for $b \leqslant \eta \leqslant \lambda b$.

Since $\varepsilon=\frac{1}{p(1-k)+(k+m+p-3)}-\alpha$ we choose $A$ such that

$$
\begin{aligned}
& \left(\frac{k+m+p-3}{p}\right)^{p-1}(\alpha+\varepsilon)< \\
& <A^{k+m+p-3}<\frac{1}{((1-k) p+(k+m+p-3))}\left(\frac{k+m+p-3}{p}\right)^{p-1}(1+(k+m+p-3) \varepsilon) .
\end{aligned}
$$

Hence, we have

$$
((1-k) p+(k+m+p-3))\left(\frac{p}{k+m+p-3}\right)^{p-1} A^{k+m+p-3}<1+(k+m+p-3) \varepsilon
$$

It implies that $g(y)$ reaches maximum at $y_{*}$, where

$$
y_{*}=\left(\frac{(k+m+p-3)^{p-1} \gamma b}{(k+m+p-3)^{p-1}+(k+m+p-3)^{p} \varepsilon-((1-k) p+(k+m+p-3)) p^{p-1} A^{k+m+p-3}}\right)^{\frac{1}{p-1}} .
$$

Therefore $g\left(\eta^{\frac{1}{p-1}}\right) \leqslant g\left(y_{*}\right)$. To prove $(22)$, we assume that $g\left(y_{*}\right) \leqslant 0$, that is,

$$
\begin{gathered}
\frac{1}{k+m+p-3}\left[A^{k+m+p-3}((1-k) p+(k+m+p-3))\left(\frac{p}{k+m+p-3}\right)^{p-1}-1-\right. \\
-(k+m+p-3) \varepsilon] y_{*}^{p}+\frac{\gamma p}{k+m+p-3} b y_{*}- \\
\quad-\left[A^{k+m+p-3}\left(\frac{p}{k+m+p-3}\right)^{p-1}-(\alpha+\varepsilon)\right](\lambda b)^{\frac{p}{p-1}} \leqslant 0 .
\end{gathered}
$$

By substituting the value of $y_{*}$ into the above inequality, we obtain

$$
\begin{aligned}
& \frac{p}{k+m+p-3} \gamma^{\frac{p}{p-1}}(1+(k+m+p-3) \varepsilon- \\
& \left.-((1-k) p+(k+m+p-3))\left(\frac{p}{k+m+p-3}\right)^{p-1} A^{k+m+p-3}\right)^{-\frac{1}{p-1}} \leqslant \\
& \leqslant\left[A^{k+m+p-3}\left(\frac{p}{k+m+p-3}\right)^{p-1}-(\alpha+\varepsilon)\right](\lambda)^{\frac{p}{p-1}} .
\end{aligned}
$$

If we choose the constant $\lambda>1$ such that 


$$
\begin{aligned}
& \lambda \geqslant\left(\frac{p}{k+m+p-3}\right)^{\frac{p-1}{p}} \gamma\left[A^{k+m+p-3}\left(\frac{p}{k+m+p-3}\right)^{p-1}-(\alpha+\varepsilon)\right]^{-\frac{p-1}{p}} \times \\
& \times\left(1+(k+m+p-3) \varepsilon-((1-k) p+(k+m+p-3))\left(\frac{p}{k+m+p-3}\right)^{p-1} A^{k+m+p-3}\right)^{-\frac{1}{p}}
\end{aligned}
$$

then $g\left(\eta^{\frac{1}{p-1}}\right) \leqslant 0$ for any $b \leqslant \eta \leqslant \lambda b$. In particular, inequality (20) holds. For fixed $A, \lambda$ we notice that $q>p(1-k)+(k+m+p-3)$. We assume that $b$ is small enough such that $(21)$ holds.

Inequality $p q-p(1-k)-(k+m+p-3)<(p-1)(\beta-1)$ implies that $-\alpha \beta+\alpha+1<0$. We can choose large enough $\tau$ to obtain

$$
\begin{gathered}
(t+\tau)^{-\alpha \beta+\alpha+1} f^{\beta}=(t+\tau)^{-\alpha \beta+\alpha+1} A^{\beta}\left((\lambda b)^{\frac{p}{p-1}}-(\xi+b)^{\frac{p}{p-1}}\right)_{+}^{\frac{\beta(p-1)}{k+m+p-3}} \leqslant \\
\leqslant(t+\tau)^{-\alpha \beta+\alpha+1} A^{\beta-1}(\lambda b)_{+}^{\frac{(\beta-1) p}{k+m+3}} f \leqslant \varepsilon f .
\end{gathered}
$$

It follows from above inequality and (18) that (17) holds. Therefore, $\bar{u}$ given by (16) and (19) is a supersolution and any solution of problem (1)-(3) with initial data $u_{0}$ lying below $\bar{u}(x, 0)=\tau^{-\alpha} f\left(x \tau^{-\gamma}\right)$, and it is global in time. Moreover, it tends to zero as $t \rightarrow \infty$.

Case 2. Let $p q-p(1-k)-(k+m+p-3) \geqslant(p-1)(\beta-1), \beta>p(1-k)+(k+m+p-2)$. We found that the global solution for $p q-p(1-k)-(k+m+p-3)<(p-1)(\beta-1)$ and $q>p(1-k)+(k+m+p-3)$ goes to zero. We can assume that it is less than one. Therefore, for every pair of exponents $(\bar{\beta}, \bar{q})$ such that $\bar{\beta} \geqslant \beta, \bar{q} \geqslant q$, it is also a supersolution to problem (1)-(3) with small initial data.

Thus we have a global nontrivial supersolution for every $p q-p(1-k)-(k+m+p-3) \geqslant$ $\geqslant(p-1)(\beta-1)$ with $\beta>p(1-k)+(k+m+p-2)$. According to the comparison principle, the solution of problem (1)-(3) is global solution if the initial data $u_{0}$ is small enough.

\section{References}

[1] A.A.Samarskii, V.A.Galaktionov, S.P.Kurdyumov, A.P.Mikhailov, Blow-Up in Quasilinear Parabolic Equations, de Gruyter Expositions in Mathematics, 1995.

[2] J.Muller, C.Kuttler, Methods and models in mathematical biology, Springer, Deterministic and stochastic approaches Berlin, 2015.

[3] C.V.Pao, Nonlinear Parabolic and Elliptic Equation, Plenum, New York, 1992.

[4] P.Li.Zhongping, L.Mu.Chunlai, Li Xie, Critical curves for a degenerate parabolic equation with multiple nonlinearities, J. Math. Anal. Appl., 359(2009), 39-47.

[5] M.Aripov, S.A.Sadullaeva, To properties of solutions to reaction-diffusion equation with double nonlinearity with distributed parameters, J. Sib. Fed. Univ. Math. Phys., 6(2013), no. $2,157-167$.

[6] Z.R.Rakhmonov, A.I.Tillaev, On the behavior of the solution of a nonlinear polytropic filtration problem with a source and multiple nonlinearities, NANOSYSTEMS: physics, chemistry, mathematics, 9(3)(2018), 323-329.

[7] Z.R.Rakhmonov, On the Properties of Solutions of Multidimensional Nonlinear Filtration Problem with Variable Density and Nonlocal Boundary Condition in the Case of Fast Diffusion, Journal of Siberian Federal University. Mathematics \& Physics, 9(2016), no 2, 225-234. 
[8] V.A.Galaktionov, J.L.Vazquez, The problem of blow-up in nonlinear parabolic equations, Discrete and continuous dynamical systems, 8(2002), no. 2, 399-433.

[9] X.Song, S.Zheng, Blow-up and blow-up rate for a reaction-diffusion model with multiple nonlinearities, Nonlinear Anal., 54(2003), 279-289.

[10] Z.Li, Ch.Mu, Critical exponents for a fast diffusive polytrophic filtration equation with nonlinear boundary flux, J. Math. Anal. Appl., 346(2008), 55-64.

[11] Zhongping Li, Chunlai Mu, Li Xie, Critical curves for a degenerate parabolic equation with multiple nonlinearities, J. Math. Anal. Appl., 359(2009), 39-47.

[12] Z.Wang, J.Yin, Ch.Wang, Critical exponents of the non-Newtonian polytropic filtration equation with nonlinear boundary condition, Appl. Math. Lett., 20(2007), 142-147

[13] J.R.Raimbekov, The properties of the solutions for Cauchy problem of nonlinear parabolic equations in non-divergent form with density, J. Sib. Fed. Univ. Math. Phys., 8(2015), no. 2, $192-200$.

[14] C.Jin, J.Yin, Critical exponent of a doubly degenerate parabolic equation in non-divergence form with nonlinear source, Chin. Ann. Math., Ser. A, 30(2009), 525-538

[15] C.Jin, J.Yin, Self-similar solutions for a class of non-divergence form equations, Nonlinear Differ. Equ. Appl. Nodea, 20(2013), no. 3, 873-893

[16] C.Wang, J.Yin, Shrinking self-similar solutions of a nonlinear diffusion equation with nondivergence form, J. Math. Anal. Appl., 289(2004), 387-404.

[17] W.Zhou, Z.Yao, Cauchy problem for a degenerate parabolic equation with non-divergence form, Acta. Mathematica Scienta, 30B(2010), no. 5, 1679-1686

[18] W.Zhou, Z.Wu, Some results on a class of degenerate parabolic equations not in divergence form, Nonlinear Analysis: Theory, Methods Applications, 60(2005), no. 5, 863-886

\section{Критические кривые параболического уравнения с двойной нелинейностью недивергентного вида с источником и нелинейным граничным потоком}

\section{Мирсаид М. Арипов \\ Национальный университет Узбекистана Университетская, 4, Ташкент, 100174 Узбекистан \\ Жахонгир Р. Раимбеков Университет Инха в городе Ташкенте Зиёлилар, 9, Ташкент, 100041 Узбекистан}

$\overline{B ~ д а н н о и ̆ ~ р а б о т е ~ и с с л е д у ю т с я ~ к р и т и ч е с к и е ~ к р и в ы е ~ у р а в н е н и я ~ с ~ д в о и ̆ н о и ̆ ~ н е л и н е и ̆ н о с т ъ ю ~ н е д и в е р-~}$ гентного вида с нелинейным граничным потоком. А именно получена критическая кривая глобального существования и критическая кривая Фуджита.

Ключевые слова: уравнение с двойной нелинейностъю недивергентного вида, критическая кривал Фуджита, критическая кривая глобального существования. 